\title{
Complications rate of and risk factors for the unplanned reoperation of degenerative lumbar spondylolisthesis in elderly patients: a retrospective single-centre cohort study of $\mathbf{3 3}$ patients
}

\section{Weiyang Zhong}

Chongqing Medical University First Affiliated Hospital

\section{Xinjie Liang}

Chongqing Medical University First Affiliated Hospital

Xiaoji Luo ( $\sim$ cy2982@163.com )

Chongqing Medical University First Affiliated Hospital

\section{Tianji Huang}

Chongqing Medical University First Affiliated Hospital

\section{Zhengxue Quan}

Chongqing Medical University First Affiliated Hospital

\section{Research article}

Keywords: degenerative lumbar spondylolisthesis, unplanned operation, risk factor, geriatric patients

Posted Date: May 1st, 2020

DOI: https://doi.org/10.21203/rs.2.18807/v2

License: (c) (1) This work is licensed under a Creative Commons Attribution 4.0 International License. Read Full License

Version of Record: A version of this preprint was published on August 24th, 2020. See the published version at https://doi.org/10.1186/s12877-020-01717-2. 


\section{Abstract}

Background: The study aimed to investigate the complications rate of and risk factors for unplanned reoperation among elderly patients who underwent posterior lumbar fusion (PLF) for degenerative lumbar spondylolisthesis (DLS).

Methods: A total of 1100 DLS patients who were older than 60 years were reviewed from January 2006 to December 2016; 33 patients underwent unplanned reoperations and were analysed and divided into two groups (group A: posterolateral fusion, 650 patients; group B: intervertebral fusion, 450 patients). Sex, body mass index (BMI), radiographic data and clinical outcome data were analysed to evaluate the complications rate of and the risk factors for unplanned reoperations.

Results: A total of 33 patients underwent unplanned reoperations (3\%). The patients were followed for an average of $4.20 \pm 2.25$ years (group A) and $4.32 \pm 2.54$ years (group B) without a significant difference. Significant differences were found in mean age, levels of involvement, hospital stay, surgery time, and blood loss between the groups. The causes of unplanned operation were wound infection, screw misplacement, neurological deficit, nonunion, and screw fracture, which were significant except for wound infection between the groups. Higher BMI (obesity), diabetes mellitus (DM), more bleeding and sex (female) were risk factors for complications. Cases of screw misplacement, neurological deficit, nonunion and screw fracture in group A were more significant than those in group $B$.

Conclusion: Patients with higher BMI, DM, older age, posterolateral fusion, and female sex predicted a higher incidence of unplanned reoperations. Spine surgeons may need to pay more attention to their preoperative training and to improving surgical techniques that could reduce the reoperation rate.

\section{Background}

With increases in the ageing population, the incidence of degenerative lumbar spondylolisthesis (DLS) and its surgical treatments has also received increasing attention because the rapid progression of anaesthesiology and surgical instruments has led to active surgical management in elderly patients[1]. However, preventing revision spinal surgery is still a matter of cardinal significance for spine surgeons and their patients. Unplanned reoperation is due to an unexpected event, the presence of persistent symptoms, the deterioration of previous potential diseases, or complications related to the primary operation[2-3]. A better understanding of the complications rate of and risk factors for unplanned reoperations may help improve surgical outcomes and prognoses. It was reported that the cumulative reoperation rate in lumbar degenerative diseases was $4.7 \%$ at 3 months, $6.1 \%$ at 1 year, $8.5 \%$ at 2 years, $15.2 \%$ at 3 years, $17.7 \%$ at 5 years and $23.3 \%$ (38/163 patients) at the final follow-up[4-8]. Reducing the rate of lumbar surgery revision is also vital because the outcomes of DLS reoperation may be worse than the results of the initial surgical management.

Therefore, the study aimed to investigate the incidence of unplanned reoperation in elderly patients who underwent surgery for DLS and to analyse the complications rate and risk factors. 


\section{Methods}

\section{Patient selection}

The study was approved by the institutional review board of our hospital. A total of 1100 patients were retrospectively reviewed, and the unexpected reasons for the unplanned reoperation were recorded and assessed. Between January 2006 and December 2016, 33 reoperation patients with DLS, aged more than 60 years old, were enrolled.

The inclusion criteria were one-level or two-level DLS requiring surgical treatment again. All the surgeries were performed for posterior lumbar fusion, including posterolateral fusion (650 patients) and intervertebral fusion (450 patients). Unplanned surgery was defined as reoperation in the operating room after the primary surgery. The primary and revision surgeries were all performed by the same senior surgical team. The exclusion criteria were as follows: patients who suffered from lumbar disc herniation, lumbar spinal stenosis, lumbar vertebral fractures, spondylitis or tumours.

Patient demographic data, including the primary surgical procedure and revision surgery information, were recorded in our study. The reasons for the unplanned surgery were assessed, and the reoperation rates of DLS were calculated.

\section{Statistical analysis}

All statistical data were analysed with SPSS version 22.0 statistical software (SPSS, Inc., Chicago, IL, USA). The data were analysed by Student's t-test for continuous variables and the Chi-square test for categorical variables. Values of $\mathrm{P}<0.05$ were regarded as statistically significant. For all regression models, a p value less than 0.05 with a confidence interval $(\mathrm{Cl})$ of $95 \%$ was considered significant.

\section{Results}

A total of 33 patients underwent unplanned reoperations (3\%). The patients were followed for an average of $4.20 \pm 2.25$ years (group A) and $4.32 \pm 2.54$ years (group B) without significant differences. Significant differences were found in the mean age, levels of involvement, hospital stay, surgery time, and blood loss between the groups (Table 1). The causes of unplanned operation were wound infection, screw misplacement (Figure 1), neurological deficit, nonunion (Figures 3, 4), and screw fracture (Figure 2), which were significant except for wound infection between the groups. Cases of screw misplacement, neurological deficit, nonunion and screw fracture in group A were more significant than those in group B.

Table 1 Comparison of the baseline data 


\begin{tabular}{clll}
\hline & Posterolateral fusion & Intervertebral fusion & P value \\
\hline Patients (n) & $24(24 / 650)$ & $9(9 / 450)$ & \\
Males/females & $9 / 15$ & $3 / 6$ & 0.583 \\
Mean age (y) & $72.80 \pm 11.7$ & $71.90 \pm 10.7$ & 0.654 \\
Follow-up (y) & $4.20 \pm 2.25$ & $4.32 \pm 2.54$ & \\
Involved levels & & & $<0.0001$ \\
L4-5 & 18 & 5 & $<0.0001$ \\
L5-S1 & 6 & 4 & $<0.0001$ \\
Hospital stay (days) & $12.55 \pm 3.35$ & $15.43 \pm 5.20$ & $<0.0001$ \\
Surgery time (minutes) & $100.50 \pm 30.50$ & $15.43 \pm 5.20$ & $<0.0001$ \\
Blood loss (ml) & $150.65 \pm 35.45$ & $230.90 \pm 101.50$ & 0.730 \\
Wound infection & 4 & 3 & $<0.0001$ \\
Screw misplacement & 7 & 3 & $<0.0001$ \\
Neurological deficit & 5 & 1 & $<0.0001$ \\
Nonunion & 4 & 1 & $<0.0001$ \\
Screw fracture & 4 & 1 & \\
\hline
\end{tabular}

Wound infections were found in patients after debridement surgery without implant removal, and these patients were treated with antibiotics sensitive to the bacteria. Pedicle screw displacement was detected on postoperative X-ray from patients who manifested leg pain or persistent sciatica after the primary surgery. All of the screws were corrected immediately; the leg pain improved after the reoperation and recovered to normal at the final follow-up.

\section{Risk factor analysis}

Based on the previous documents, the potential factors were selected and assessed for analysing risk factors using the following data: age, sex, body mass index (BMI), and fusion surgical methods. Higher $\mathrm{BMI}$, diabetes mellitus (DM), more bleeding and female sex predicted a higher occurrence of wound infection (Table 2). Different fusion methods and female sex predicted the development of nonunion and screw fracture.

Table 2 Risk analysis for wound infection 


\begin{tabular}{lllll}
\hline Valuables & OR & P value & \multicolumn{2}{l}{ 95\% confidence interval } \\
\hline BMI (obesity) & 4.42 & 0.01 & 1.5 & 14.06 \\
Sex (female) & 3.82 & 0.015 & 1.05 & 9.73 \\
Diabetes mellitus & 3.08 & 0.025 & 0.78 & 7.85 \\
Age & 3.65 & 0.02 & 0.96 & 1.45 \\
Fusion method & 4.02 & 0.010 & 2.01 & 16.21 \\
More bleeding & 3.01 & 0.03 & 0.65 & 7.21 \\
\hline
\end{tabular}

\section{Discussion}

With the increase in the elderly population in China, an increasing number of aged patients with degenerative lumbar disease (DLD) require surgical treatment. DLS is a type of spondylolisthesis with an intact neural arch. The treatment objectives of surgery include neural decompression, motion segment stabilization, intervertebral disc height reconstruction and sagittal balance restoration[1-2][9-12]. Spondylolytic segment stabilization relies on fusion through an anterior, a posterior or a combined approach[12-13]. The PLF technique has gained reliability and popularity for DLS. Posterolateral fusion or intervertebral interbody fusion immediately and rapidly reconstructs a biomechanically stable spine, thereby increasing the chance of fusion[14-16]. However, in elderly patients, it is a challenge to perform spine surgery because of the increasing presence of medical diseases and surgical complications[16]. Furthermore, reoperation is an unplanned event for these patients, their families and surgeons, which results in additional perioperative complications, including death and potential medical risks or medical disputes. It is important for surgeons to identify risk factors in order to make better preoperative decisions and evaluate the surgical procedures to avoid unplanned reoperations.

Several previous studies reported reoperation following primary lumbar surgery for degenerative conditions and indicated that the revision rates were $14.0 \%$ in the 1997 to 2000 cohort and $12.4 \%$ in the 1990 to 1993 cohort[17]. Ghogawala demonstrated that the reoperation rate after only decompression for DLS at 1 year postoperatively was $15 \%[18]$, while Blumenthal reported a rate of $37.5 \%$ at a mean followup of 3.6 years[19]. The reoperation rate found in the present study was nearly the same as that in previous studies: the reoperation rate for only decompression at the 1-year follow-up was $10.8 \%$, and the rate increased to $29.7 \%$ at 5 years postoperatively and to $33.4 \%$ at the final follow-up[20-21]. Another report demonstrated that the cumulative reoperation rate was $6.1 \%$ at 1 year, $8.5 \%$ at 2 years, $15.2 \%$ at 3 years, $17.7 \%$ at 5 years, and $23.3 \%$ at the final follow-up[22]. In contrast to the studies mentioned above, in our study, the reoperation rate of DLS was $3.0 \%$, which was lower than the rates found in previous studies. The reason for the lower reoperation rate in our study may be that we only reported reoperation during patients with one-level or two-level DLS.

Elderly patients have commonly been considered to be at a higher risk of postoperative complications from DLS than younger patients. An approximately $80 \%$ complications rate has been document in patient who undergo surgery for DLS[23-27]. In the study by Okuda, postoperative complications were found in 
$16 \%$ of elderly patients after PLIF with pedicle screw placement[28]. In our series, the major reasons for reoperation were wound infection, screw misplacement, neurological deficit, nonunion, and screw fracture, which were similar to previous reports. Therefore, a better understanding of the complications, risk factors and unplanned reoperation rate is helpful for improve surgical outcomes.

In our study, higher BMI, DM, more bleeding and female sex predicted the occurrence of wound infection, which other studies have previously reported. Deep postoperative wound infection will significantly impact the surgical outcomes of DLS and will have an important influence on the surgical plan, which requires revision surgery with implant removal if necessary. This complication also increases the cost of hospitalization and nursing. Additionally, spine surgeons should pay more attention to improving surgical training and may need more help from multiple disciplinary teams (MDTs).

Another common reason for unplanned reoperation was screw displacement in the study. Complications related to screw displacement in spinal surgery are common and often result in spinal cord and nerve root injuries as well as dural lesions that require immediate revision surgery. Fortunately, all neurological defects in the patients after reoperation were alleviated and returned to normal at the final follow-up. Additional spine courses or navigation systems may help reduce the rate of screw displacement. Additionally, we found that patients who underwent posterolateral fusion procedures had a higher incidence of nonunion and a higher rate of unplanned reoperation. The results showed that a detailed preoperative plan and strict follow-up may work for patients with DLS when indicated for surgical treatment.

\section{Conclusion}

The unplanned reoperation rate in elderly patients who underwent surgery for DLS was $3 \%$. Patients with higher BMI, DM, older age, posterolateral fusion, and female sex predicted a higher incidence of unplanned reoperations. Spine surgeons may need to pay more attention to their preoperative training and to improving the surgical techniques of DLS treatment, which could reduce the reoperation rate. However, we want to point out that there are several limitations in this study. First, the study did not consider the distribution of one-level or two-level DLS in each group, which was associated with bias. Second, the retrospective nature of the small-sample study may be associated with bias. In the future, prospective, randomized studies with long-term follow-up periods are needed.

\section{Abbreviations}

PLF: posterior lumbar fusion; DLS: degenerative lumbar spondylolisthesis; BMI: body mass index; DM: diabetes mellitus; DLD: degenerative lumbar disease; MDT: multiple disciplinary team

\section{Declarations}

\section{Ethics approval and consent to participate}


The Institutional Review Board of the First Affiliated Hospital of Chongqing Medical University approved this study. The written informed consent was obtained from all participants.

\section{Consent for publication}

Not applicable.

\section{Availability of data and materials}

The datasets generated and/or analysed during the current study are not publicly available because the data are confidential patient data, but they are available from the corresponding author upon reasonable request.

\section{Competing interests}

The authors declare that they have no competing interests.

\section{Funding}

The authors declare no sources of funding.

\section{Authors' contributions}

Contributions: (I) conception and design: WYZ and XJiL; (II) administrative support: XJieL and ZXQ; (III) provision of study materials or patients: $\mathrm{WYZ}, \mathrm{XJiL}, \mathrm{TJH}$, and ZXQ; (IV) collection and assembly of data: WYZ and XJieL; and (V) data analysis and interpretation: WYZ, TJH, and XJieL. All authors read and approved the final manuscript.

\section{Acknowledgements}

Not applicable.

\section{Author details}

${ }^{1}$ Department of Orthopedic Surgery, The First Affiliated Hospital of Chongqing Medical University, Chongqing, China

${ }^{2}$ Department of Pain Management, The First Affiliated Hospital of Chongqing Medical University, Chongqing, China

* Correspondence: cy2982@163.com

\section{References}


1. Matz PG, Meagher RJ, Lamer T, et al. Guideline summary review: an evidence-based clinical guideline for the diagnosis and treatment of degenerative lumbar spondylolisthesis. Spine J. 2016;16(3):43948.

2. Jacobsen $\mathrm{S}$, Sonne-Holm S, Rovsing $\mathrm{H}$,et al.Degenerative lumbar spondylolisthesis: an epidemiological perspective: the Copenhagen Osteoarthritis Study. Spine (Phila Pa1976). 2007;32(1):120-5.

3. Sclafani JA, Constantin A, Ho PS, et al. Descriptive analysis of spinal neuroaxial injections, surgical interventions, and physical therapy utilization for degenerative lumbar spondylolisthesis within medicare beneficiaries from 2000 to 2011. Spine (Phila Pa 1976). 2017;42(4):240-6.

4. Gerling MC, Leven D, Passias PG,et al. Risks factors for reoperation in patients treated surgically for degenerative spondylolisthesis: a subanalysis of the 8 year data from the SPORT trial. Spine (Phila Pa 1976). 2017,42(20):1559-1569.

5. Forsth $\mathrm{P}$, Olafsson $\mathrm{G}$, Carlsson $\mathrm{T}$, et al. A randomized, controlled trial of fusion surgery for lumbar spinal stenosis. N Engl J Med. 2016;374(15):1413-23.

6. Weinstein JN, Lurie JD, Tosteson TD, et al. Surgical versus nonsurgical treatment for lumbar degenerative spondylolisthesis. N Engl J Med. 2007;356(22): 2257-70.

7. Ghogawala Z, Dziura J, Butler WE, et al. Laminectomy plus fusion versus laminectomy alone for lumbar spondylolisthesis. N Engl J Med. 2016;374(15):1424-34.

8. Challier V, Boissiere L, Obeid I, et al. One-level lumbar degenerative spondylolisthesis and posterior approach: is transforaminal lateral interbody fusion mandatory?A randomized controlled trial with 2year follow-up. Spine(Phila Pa 1976). 2017;42(8):531-9.

9. Isaacs RE, Sembrano JN, Tohmeh AG, Group SDS. Two-year comparative outcomes of MIS lateral and MIS transforaminal interbody fusion in the treatment of degenerative spondylolisthesis. Part II: radiographic findings. Spine (Phila Pa 1976). 2016;41(Suppl 8):S133-44.

10. Sembrano JN, Tohmeh A, Isaacs R, Group SDS. Two-year comparative outcomes of MIS lateral and MIS transforaminal interbody fusion in the treatment of degenerative spondylolisthesis. Part I: clinical findings. Spine (Phila Pa 1976). 2016;41(Suppl 8):S123-32.

11. Matsunaga S, ljiri K, Hayashi K. Nonsurgically managed patients with degenerative spondylolisthesis: a 10- to 18-year follow-up study. J Neurosurg. 2000;93(2 Suppl):194-8.

12. Pearson AM, Lurie JD, Blood EA, et al. Spine patient outcomes research trial: radiographic predictors of clinical outcomes after operative or nonoperative treatment of degenerative spondylolisthesis. Spine (Phila Pa 1976).2008;33(25):2759-66.

13. Musluman AM, Cansever T,et al. Midterm outcome after a microsurgical unilateral approach for bilateral decompression of lumbar degenerative spondylolisthesis. J Neurosurg Spine. 2012;16(1):68-76.

14. Aihara T, Toyone T, Aoki Y, et al. Surgical management of degenerative lumbar spondylolisthesis: a comparative study of outcomes following decompression with fusion and microendoscopic decompression.J Musculoskelet Res. 2012;15(4):1250020. 
15. Inui T, Murakami M, Nagao N, et al. Lumbar degenerative spondylolisthesis: changes in surgical indications and comparison of instrumented fusion with two surgical decompression procedures. Spine (Phila Pa 1976). 2017;42(1):E15-24.

16. Ahmad S, Hamad A, Bhalla A, et al. The outcome of decompression alone for lumbar spinal stenosis with degenerative spondylolisthesis. Eur Spine J. 2017;26(2):414-9.

17. Mardjetko SM, Connolly PJ, Shott S. Degenerative lumbar spondylolisthesis. A meta-analysis of literature 1970-1993. Spine(Phila Pa 1976). 1994;19(20 Suppl):2256S-65S.

18. Ghogawala Z, Benzel EC, Amin-Hanjani S, et al. Prospective outcomes evaluation after decompression with or without instrumented fusion for lumbar stenosis and degenerative Grade I spondylolisthesis. J Neurosurg Spine 2004;1:267-72.

19. Blumenthal C, Curran J, Benzel EC, et al. Radiographic predictors of delayed instability following decompression without fusion for degenerative grade I lumbar spondylolisthesis. J Neurosurg Spine 2013;18:340-6.

20. Martin CR, Gruszczynski AT, Braunsfurth HA, et al. The surgical management of degenerative lumbar spondylolisthesis: a systematic review. Spine (Phila Pa 1976).2007;32(16):1791-8.

21. Rousseau MA, Lazennec JY, Bass EC, et al. Predictors of outcomes after posterior decompression and fusion in degenerative spondylolisthesis. Eur Spine J. 2005;14(1):55-60.

22. Shunsuke Sato, Mitsuru Yagi, Masayoshi Machida et al.Reoperation rate and risk factors of elective spinal surgery for degenerative spondylolisthesis: minimum 5-year follow-up .The Spine Journal, 2015,15:1536-1544 .

23. Liu J-M, Deng H-L, Peng A-F, et al. Unplanned reoperation of lumbar spinal surgery during the primary admission. Spine (Phila Pa 1976) 2016;41:1279-83 .

24. Núñez-Pereira $S 1$,Pellisé $F 1$,Rodríguez-Pardo $D$, et al. Implant survival after deep infection of an instrumented spinal fusion. Bone Joint J 2013;95-B:1121-6.

25. Kim CH, Chung CK, Park CS, et al. Reoperation rate after surgery for lumbar spinal stenosis without spondylolisthesis: a nationwide cohort study. Spine J 2013;13:1230-7.

26. Schuster JM, Rechtine G, Norvell DC, et al. The influence of perioperative risk factors and therapeutic interventions on infec- tion rates after spine surgery: a systematic review. Spine (Phila Pa 1976) 2010;35:S125-37.

27. Deyo RA. Trends, major medical complications, and charges associated with surgery for lumbar spinal stenosis in older adults. JAMA 2010;303:1259-65.

28. Okuda S, Iwasaki M, Miyauchi A, et al. Risk factors for adjacent segment degeneration after PLIF. Spine 2004;29:1535-40.

\section{Figures}




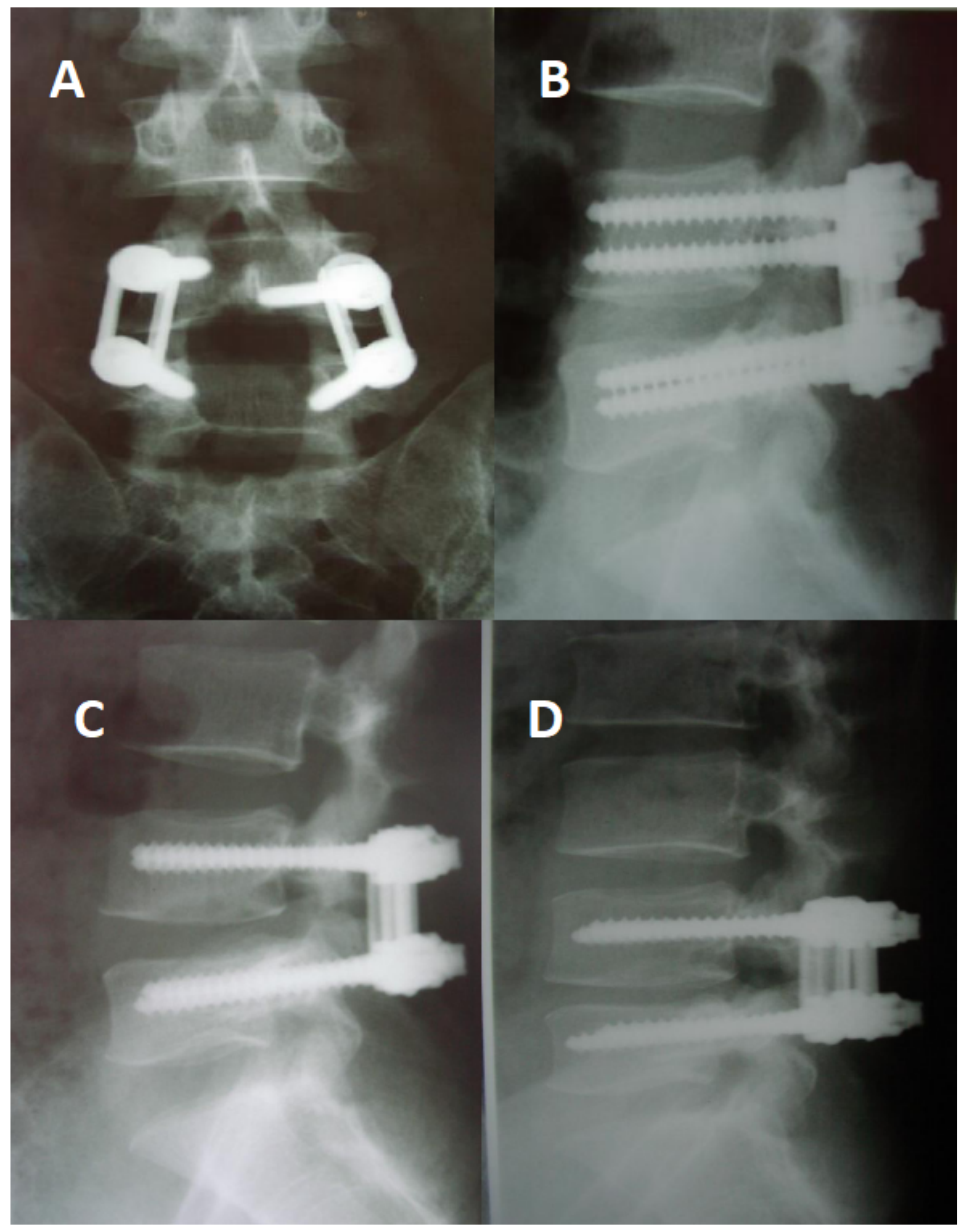

\section{Figure 1}

L4 DLS of a 65-year-old woman (BMI 32.3) treated with L4 laminectomy and posteriolateral fusion. Because of leg pain, she had revision (AB) Postoperative X-ray showed the screw displacement. (C) Postoperative X-ray showed the screw was corrected and the pain improved. (D) L4-L5 disc height narrowing and the posteriolateral fusion was observed at the 48 months' follow-up. 


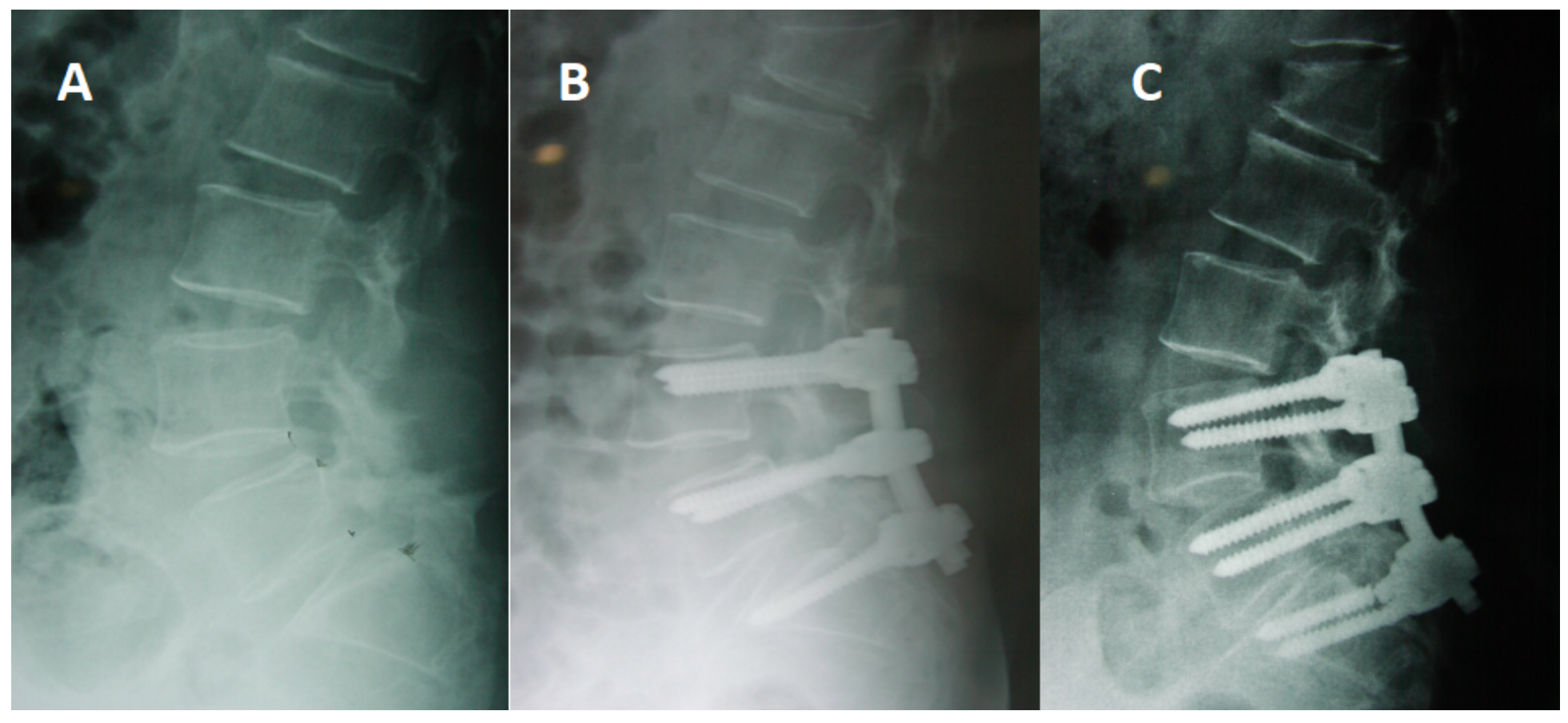

Figure 2

L4 DLS of a 72-year-old man treated with L5 laminectomy and posteriolateral fusion. (AB) Postoperative X-ray was normal. (C) The X-ray showed the screws displacement and fracture at the 36 months' followup.

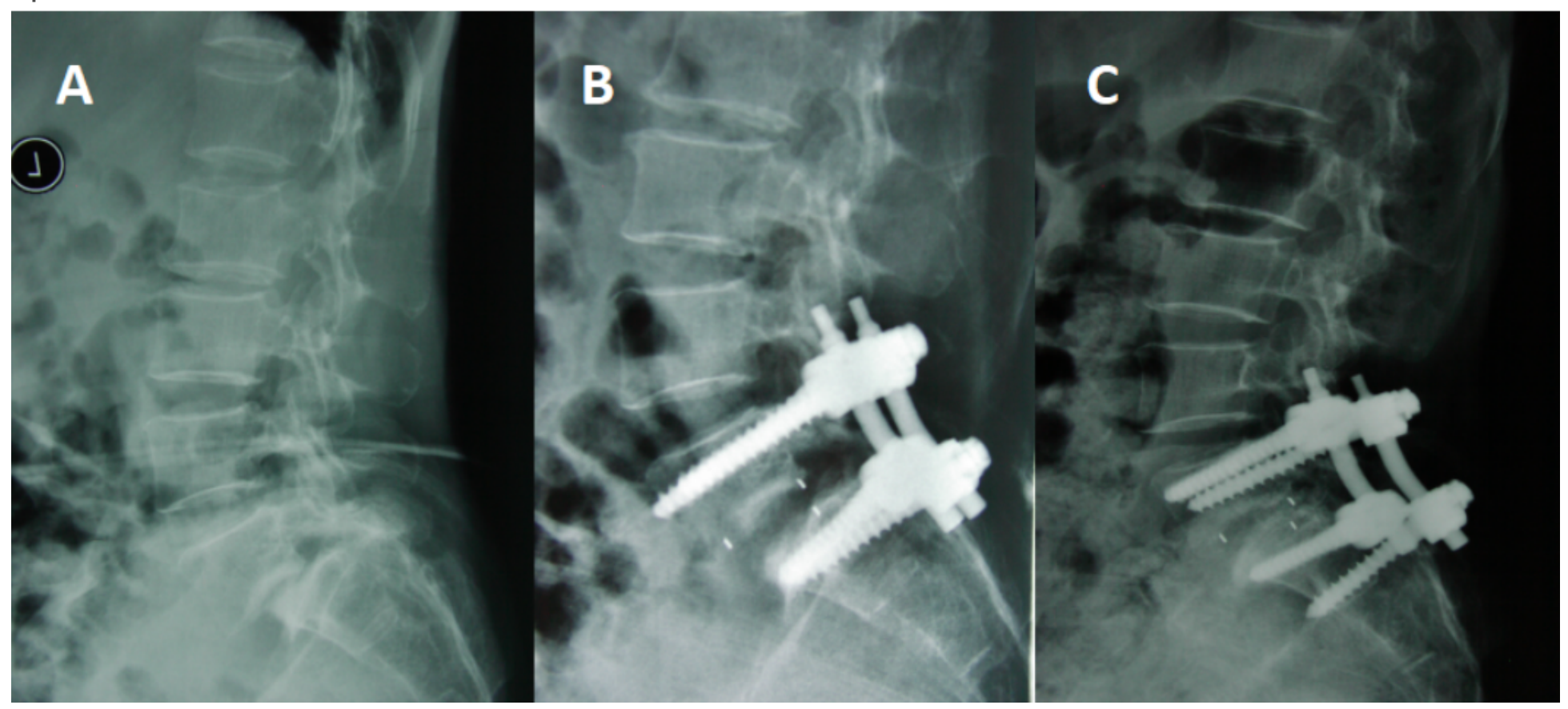

\section{Figure 3}

L5 DLS of a 75-year-old woman treated with L5 laminectomy and intervertebral interbody fusion. (AB) Postoperative X-ray was normal. (C) The X-ray showed the screws displacement and bone union at the 52-months follow-up. 


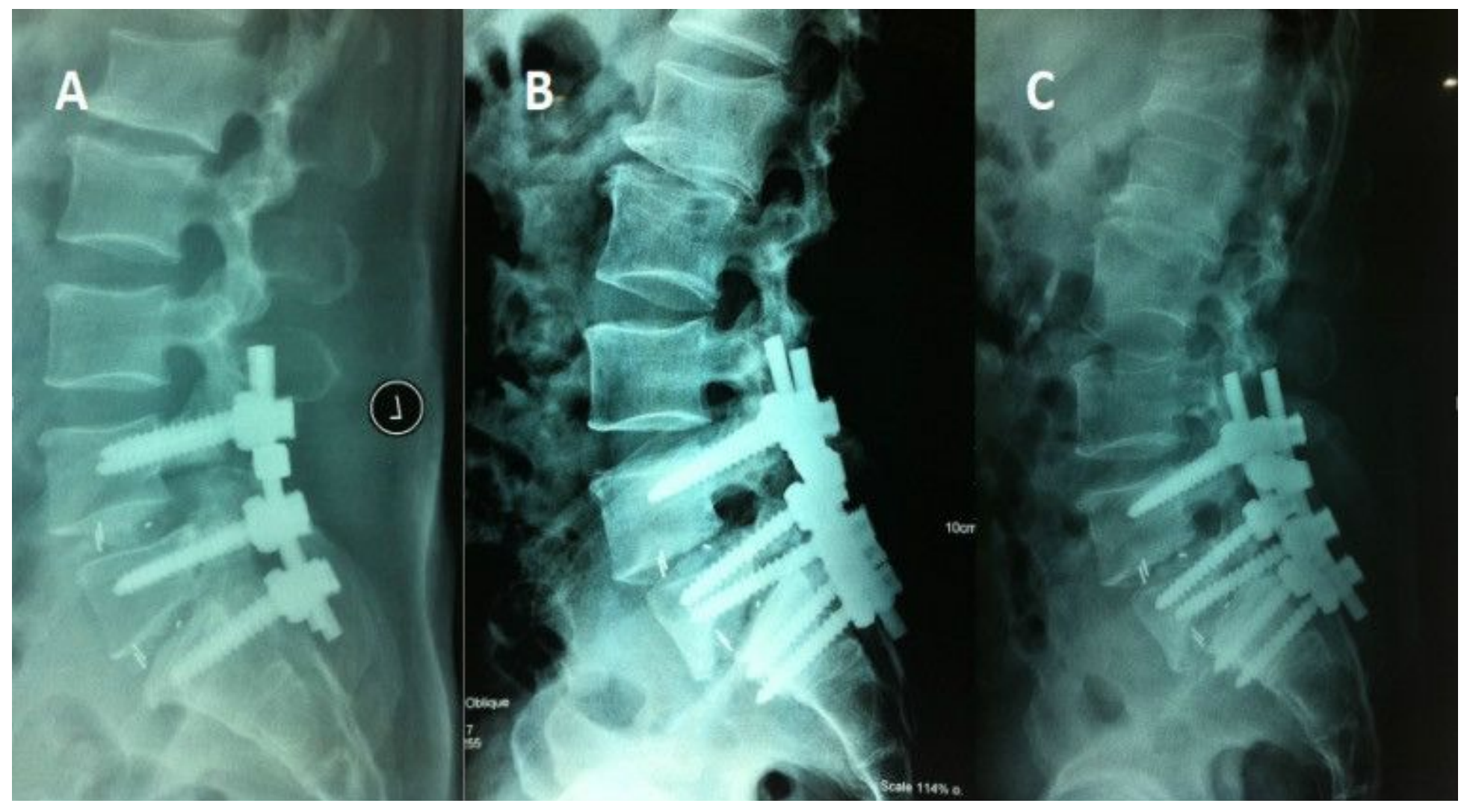

Figure 4

L4 and L5 DLS of a 75-year-old woman treated with L4/5 laminectomy and intervertebral interbody fusion(L4-5, L5-S1). (A囚The postoperative X-ray was normal. (B) The X-ray showed the bone union and screw displacement, the patient refused the revision surgery at the 12 months' follow-up. (C) The X-ray showed the screws displacement and bone union worsen at the 2-years follow-up. The patient suffered leg pain and she accepted the reoperation. 